\title{
ANTIPROLIFERATIVE AND ANTIBACTERIAL ACTIVITY OF 3-ARYLBENZO $[g]$ INDAZOLES FUNCTIONALIZED WITH NITRO AND AMINO GROUPS AT POSITION 6
}

\section{Viviana Cuartas* 1,2, María del Pilar Crespo ${ }^{3}$, Eva-María Priego ${ }^{4}$, Leentje Persoons ${ }^{5}$, Dirk Daelemans 5 , María-José Camarasa ${ }^{4}$, Braulio Insuasty ${ }^{1,2}$}

\section{and María-Jesús Pérez-Pérez ${ }^{4}$}

${ }^{1}$ Grupo de Investigación de Compuestos Heterocíclicos, Departamento de Química, Universidad del Valle, A. A. 25360 Cali, Colombia. ${ }^{2}$ Centre for Bioinformatics and Photonics-CIBioFl Calle 13 No. 100-00, Edificio E20, No. 1069, Cali, Colombia. ${ }^{3}$ Grupo de Biotecnología e Infecciones Bacterianas, Departamento de Microbiología, Universidad del Valle, Cali, Colombia. ${ }^{4}$ Instituto de Química Médica (IQM-CSIC), Juan de la Cierva 3, 28006-Madrid, Spain. ${ }^{5}$ KU Leuven Department of Microbiology, Immunology and Transplantation, Laboratory of Virology and Chemotherapy, Rega Institute for Medical Research, KU Leuven, Herestraat 49, 3000 Leuven, Belgium.

\section{INTRODUCTION}

Colchicine-site inhibitors based on combretastatin A-4 (CA-4) have been extensively studied as antimitotic and vascular disrupting agents [1]. Among the different approaches, the design and synthesis of conformationally restricted ligands have led to very potent inhibitors

Indazoles are benzo-fused pyrazoles for which a broad range of biological properties have been described, including antiproliferative and antibacterial activities $[2,3]$. These fused tricyclic pyrazole derivatives are characterized by their conformational restriction.

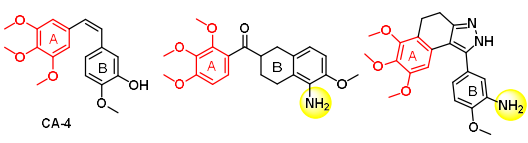

\section{OBJETIVES}

As part of our ongoing project on conformationally restricted ligands interacting at the colchicine binding site in tubulin [1], we have here designed and synthesized a novel series of tricyclic pyrazoline derivatives incorporating a nitro or an amino group at position 6 on the benzo[g]indazol ring and different aryl groups at position 3 .

\section{SYNTHESIS OF 3-ARYLBENZO[g]INDAZOLES}
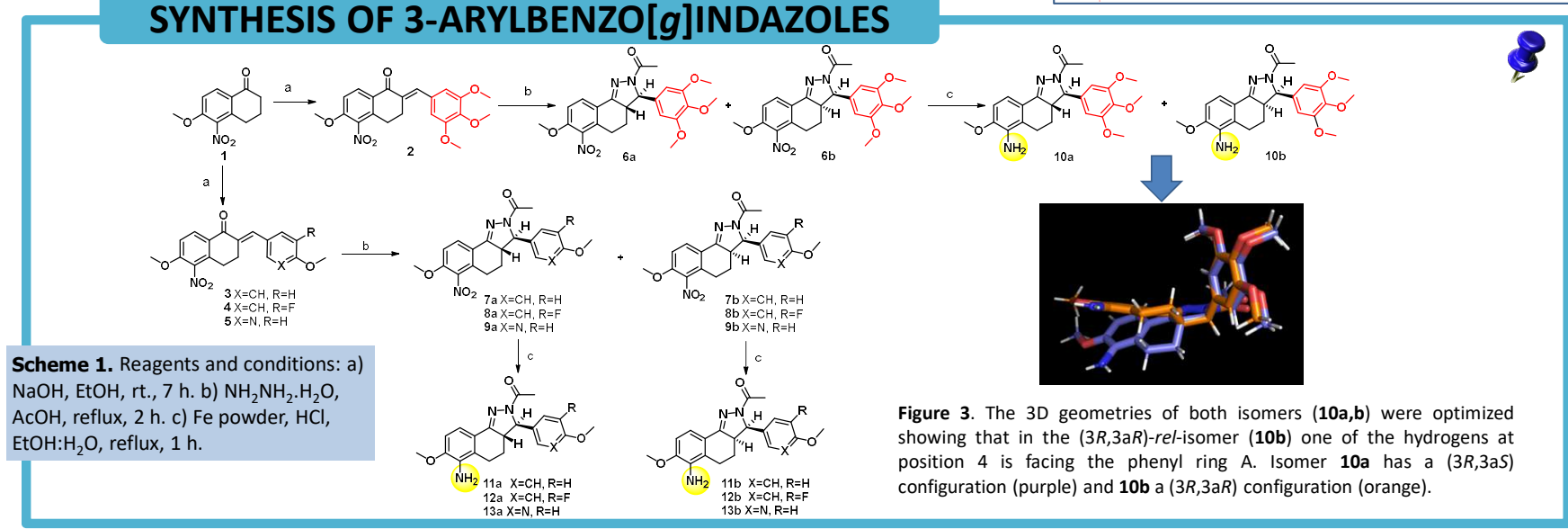

\section{ANTIPROLIFERATIVE ACTIVITY}

Table 1. Antiproliferative activity expressed in $\mathrm{IC}_{50}(\mu \mathrm{M})$

\begin{tabular}{|c|c|c|c|c|c|}
\hline & Capan-1 & Hap1 & HCT-116 & $\mathrm{NCl}-\mathrm{H} 460$ & HL-60 \\
\hline $6 a$ & $57.8 \pm 2.4$ & $62.3 \pm 4.7$ & $82.0 \pm 9.0$ & $43.7 \pm 4.7$ & $28.6 \pm 35.7$ \\
\hline $6 b$ & $42.9 \pm 12.3$ & $61.0 \pm 6.6$ & $>100$ & $44.6 \pm 0.8$ & $26.8 \pm 36.6$ \\
\hline $7 a$ & $40.8 \pm 5.0$ & $39.3 \pm 4.8$ & $29.9 \pm 9.0$ & $10.8 \pm 1.6$ & $30.9 \pm 14.8$ \\
\hline $7 \mathrm{~b}$ & $27.0 \pm 4.0$ & $37.0 \pm 2.7$ & $25.3 \pm 18.9$ & $11.8 \pm 1.6$ & $38.0 \pm 10.1$ \\
\hline $8 a$ & $42.9 \pm 1.6$ & $24.9 \pm 11.8$ & $26.9 \pm 4.8$ & $5.4 \pm 0.5$ & $42.5 \pm 0.5$ \\
\hline $8 b$ & $17.9 \pm 3.5$ & $38.5 \pm 4.1$ & $36.0 \pm 6.8$ & $14.9 \pm 2.9$ & $38.9 \pm 2.2$ \\
\hline $9 a$ & $73.4 \pm 26.6$ & $38.9 \pm 0.4$ & $36.5 \pm 1.2$ & $30.9 \pm 10.0$ & $42.7 \pm 3.9$ \\
\hline $9 b$ & $32.3 \pm 11.1$ & $41.7 \pm 3.9$ & $48.9 \pm 6.8$ & $45.0 \pm 1.7$ & $42.9 \pm 1.9$ \\
\hline $10 a$ & $30.3 \pm 9.0$ & $46.2 \pm 2.7$ & $45.2 \pm 2.6$ & $36.6 \pm 10.4$ & $52.5 \pm 16.3$ \\
\hline $10 \mathrm{~b}$ & $32.7 \pm 22.0$ & $36.4 \pm 6.6$ & $52.1 \pm 15.3$ & $44.4 \pm 2.2$ & $41.6 \pm 5.0$ \\
\hline $11 a$ & $45.1 \pm 20.6$ & $39.2 \pm 7.3$ & $43.5 \pm 6.0$ & $42.1 \pm 7.5$ & $55.9 \pm 20.8$ \\
\hline $11 b$ & $53.5 \pm 18.8$ & $52.8 \pm 7.8$ & $>100$ & $54.1 \pm 4.6$ & $44.8 \pm 27.6$ \\
\hline $12 a$ & $36.7 \pm 14.4$ & $42.6 \pm 17.7$ & $45.0 \pm 11.5$ & $39.3 \pm 8.5$ & $38.7 \pm 7.5$ \\
\hline $12 \mathrm{~b}$ & $42.6 \pm 3.9$ & $42.6 \pm 20.3$ & $66.1 \pm 31.3$ & $33.7 \pm 10.88$ & $65.9 \pm 20.2$ \\
\hline $13 a$ & $56.4 \pm 11.6$ & $80.6 \pm 9.7$ & $62.2 \pm 10.9$ & $47.3 \pm 15.3$ & $49.0 \pm 25.5$ \\
\hline $13 b$ & $60.7 \pm 1.7$ & $54.9 \pm 5.0$ & $80.6 \pm 9.7$ & $43.9 \pm 9.6$ & $65.8 \pm 17.1$ \\
\hline Docetaxel (nM) & $4.2 \pm 2.1$ & $2.3 \pm 0.7$ & $0.9 \pm 0.8$ & $3.8 \pm 2.9$ & $2.3 \pm 0.3$ \\
\hline Stauroporine (nM) & $0.7 \pm 0.2$ & $0.4 \pm 0.1$ & 0.10 & $1.5 \pm 0.4$ & $7.4 \pm 1.7$ \\
\hline
\end{tabular}

Capan-1: pancreatic adenocarcinoma; Hap1: chronic myeloid leukemia; HCT-116: colorectal carcinoma; $\mathrm{NCl}-\mathrm{H} 460$ : lung carcinoma; $\mathrm{HL}-60$ : acute myeloid leukemia. ${ }^{*} \mathrm{C}_{50}$ : Concentration of each compound that inhibits $50 \%$ of cell proliferation.

\section{Acknowledgments

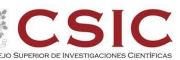 \\ KU LEUVEN

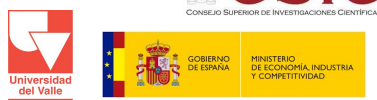 \\ (19) COLCIENCIAS}

Figure 1. Combretastatin A-4 and cis-restricted analogues. 